\title{
The gravitational wave signal from close galaxy pairs
}

\author{
Jinzhong Liu and Yu Zhang \\ National Astronomical Observatory/Xinjiang Observatory, Chinese Academy of Sciences, 150 \\ Science 1-Street, Urumqi, Xinjiang 830011, China \\ email: liujinzh@xao.ac.cn
}

\begin{abstract}
The early phase of coalescence of supermassive black hole binaries (SMBHBs) from their host galaxies provides a guaranteed source of low-frequency gravitational wave (GW) radiation by pulsar timing observations. Nowadays, SMBHBs are ubiquitous in the nuclei of galaxies. A latest sample of close galaxy pairs has been released from the Sloan Digital Sky Survey (SDSS) Data. A binary population synthesis (BPS) approach has been applied to study the characteristics of clusters and galaxies. Here we report how BPS, using SDSS results, can be used to determine the GW radiation from SMBHBs. In this study we show numerical results under the assumption that SMBHBs formed through the merger of two galaxies and give the waveform evolution using post-Newtonian approximation methods. Based on the sensitivity of the International Pulsar Timing Array (IPTA) and Square Kilometer Array (SKA) detectors, we show that the value of strain amplitude $h$ can be changed from about $10^{-14}$ to $10^{-15}$ during the observation of 20 years, which can be considered as a precise evolution.
\end{abstract}

Keywords. gravitational wave, black hole, galaxy.

\section{Introduction}

The various frequency ranges of the GW detectors can respectively observe different GW sources (Jaffe \& Backer 2003; Belczynski, Kalogera \& Bulik 2002; Liu 2009; Liu et al. 2010a; Liu et al. 2010b; Liu et al. 2012, Liu et al. 2014). The supermassive black hole binaries (SMBHBs) are thought to be the LISA verification binary GW sources. This paper provides new detailed specifics and characteristics of the SMBHBs with the latest findings of Liu et al. (2012).

\section{Computations}

Few studies can accurately determine galaxy pairs internal compositions and kinematics equations. The BL Lacertae object OJ287, will allow us to investigate a precise orbital evolution due to GW radiation in our BPS simulations, where GW radiation energy loss is appeared in the galaxy pairs. To systematically investigate the GW radiation of galaxy pair systems, we perform a Monte Carlo simulation where we follow the evolution of a sample of 1 million binaries. The properties of the overall galaxy pair sample have been displayed by the released SDSS data, including projected and angular separation, relative velocities, stellar mass ratio and the redshift. We present polynomial curve fitting formulae, which is to construct the distribution function of galaxy pairs, that approximate the evolution of SMBHBs for a wide range of mass and stellar environment. And the star formation rate is taken to be constant in the simulation. According to the derivation of black hole mass function at low redshift, a simple polynomial curve fitting approximation of SBHMF is also used. In addition to all aspects of SDSS data, the accretion disk model 

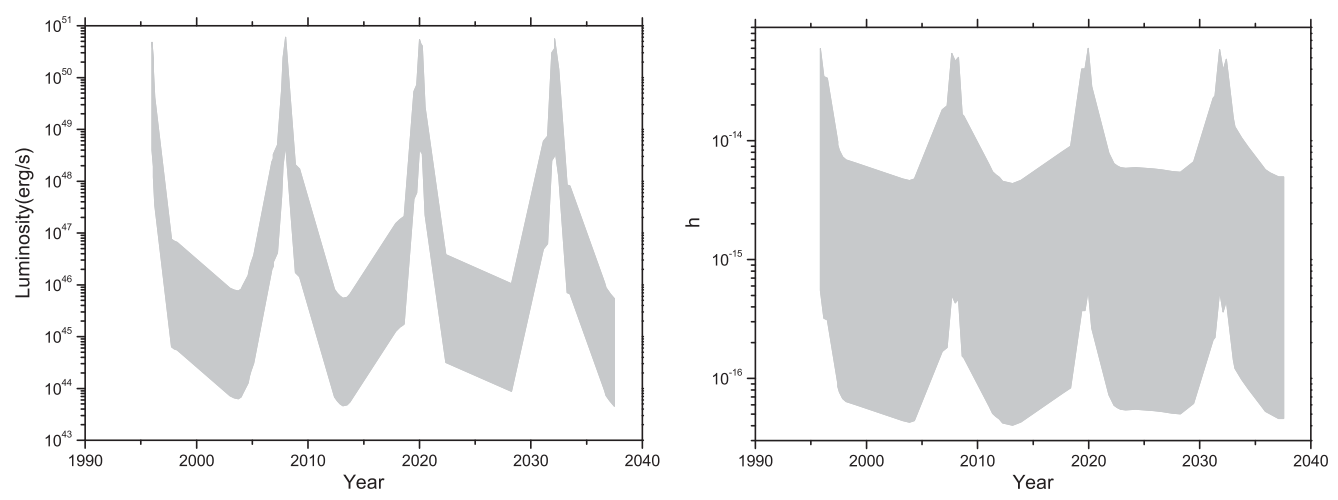

Figure 1. The strain amplitude $h$ and luminosity $L$ of GW radiation variation with time.

of primary galaxy is assumed by the analytical fitting function. And for the eccentricity of the MBHB samples, we obtain it from the random number.

\section{Results and discussion}

Figure 1 shows that the range of strain amplitude and luminosity can be covered by GW radiation from MBHBs in our Monte Carlo simulations. From these two Figures we find that there is major double-peaked GW radiation outburst at approximately 12 year intervals, which is consistent with the light curves of OJ 287. This is because that we adopt a precise orbital evolution of OJ 287 as our orbital evolutionary model to investigate the orbital track changes due to GW radiation in the BPS simulations. In other words, according to the proposal of accretion disk model, the maximum outbursts of $\mathrm{GW}$ radiation and the optical outburst are happening at the same time when the secondary BH crashes into the disk of the primary BH. From the right panel, we see that the strain amplitude of GW radiation will be detected by the SKA detector. From the left panel, we can see that our calculated MBHB samples can radiate a high luminosity due to GWs, which possibly means the influence of GW dominates the total energy loss of the galaxy pairs in the phase of coalescence. Meanwhile this variation of strain amplitude or luminosity with time maybe provides an indirect evidence for GWs existence. Especially using 20 years of observational time the pulsar timing measurements will confirm a period variation of $12 \mathrm{yr}$ in residual data. Finally, we can find some irregular "sawtooth" appearance at the maxima, which indicates that the precession of orbital period due to GW radiation and the accretion disk can influence the evolution of galaxy pairs.

\section{Acknowledgements}

This work is supported by the program of the light in China's Western Region (LCWR) (No. XBBS201221) and Natural Science Foundation (No. 11303080).

\section{References}

Belczynski, K., Kalogera, V., \& Bulik, T. 2002, ApJ, 572, 407

Jaffe, A. H. \& Backer, D. C. 2003, ApJ, 583, 616

Liu, J. Z. 2009, MNRAS, 400, 1850

Liu, J. Z., Han, Z., Zhang, F., \& Zhang, Y. 2010a, ApJ, 719, 1546

Liu, J. Z., Zhang, Y., Han, Z., \& Zhang, F. 2010b, Ap\&SS, 329, 297

Liu, J. Z., Zhang, Y., Zhang, H., Sun, Y., \& Wang, N. 2012, A\&A, 540, 67

Liu, J. Z. \& Zhang, Y. 2014, PASP, 126, 211 